\title{
Advances of Upconversion Nanoparticles for Molecular Imaging
}

Na Zhou, Jian Ni, Rong He*

Institute of Nano Biomedicine and Engineering, Key Laboratory for Thin Film and Microfabrication Technology of the Ministry of Education, Research Institute of Micro/Nano Science and Technology, Key Laboratory for the Genetics of Developmental \& Neuropsychiatric Disorders of Ministry of Education, Bio-X Center, Shanghai Jiao Tong University, Dongchuan Road 800, 200240 Shanghai, P. R. China

* Corresponding author: R. He (rhe@sjtu.edu.cn)

\begin{abstract}
Molecular imaging is developing fast towards multi-modality and simultaneous therapy. For molecular imaging, upconversion nanoparticles (UCNPs), especially lanthanide-doped nanocrystals own obvious advantages such as low toxicity, large Stokes shifts, high resistance to photo-bleaching and photochemical degradation. Moreover, near infrared (NIR) excitation contributes to the auto-fluorescence minimization, a larger penetrating depth, and less harmfulness to cells compared with traditional ultraviolet (UV) excitation. On the other hand, the composites of UCNPs with biological target molecules exert superior performance, broadening their biological application scope from multi-modality imaging, to simultaneous drug delivery and targeted therapy. Herein, we review main advances of UCNPs applied to tumor multi-modality imaging and simultaneous therapy over the past few years, explore their application prospects, and discuss the concepts, issues, approaches, and challenges, with the aim of improving the application of UCNPs in biomedical imaging and therapy in near future.
\end{abstract}

Keywords: Upconversion nanoparticles, Lanthanide-doped nanocrystals, Molecular imaging, Targeted therapy

Citation: N. Zhou et al. Advances of Upconversion Nanoparticles for Molecular Imaging. 2013, 5(3), $137-145$. DOI: 10.5101/nbe.v5i3.p137-145.

\section{Introduction}

Molecular Imaging, as an interdisciplinary, quickdeveloping area, realizes the visualization and qualitative and quantification analysis of the cellular function and gene or protein expression in living organisms. Molecular imaging exhibits great potential in applications such as the early diagnosis of diseases such as cancer, and neurological and cardiovascular diseases, and improving the treatment of these disorders by optimizing the pre-clinical and clinical tests of new medicine, as well as earlier and more precise location. Molecular and functional imaging has become a new interdisciplinary frontier in material science and clinical medicine.

Molecular imaging differs from traditional imaging in that probes known as biomarkers are used to help image particular targets or pathways. Biomarkers interact chemically with their surroundings and in turn alter the image according to molecular changes occurring within the area of interest. This process is markedly different from previous methods of imaging which primarily imaged differences in qualities such as density or water content. This ability to image fine molecular changes opens up an incredible number of exciting possibilities for medical application, including early detection and treatment of early cancer and basic pharmaceutical development. Furthermore, molecular imaging allows for quantitative tests, imparting a greater degree of objectivity to the study of these areas.

In recent years, as the nanomaterials with novel properties are fabricated lot, molecular imaging technology has made great advances. For example, fluorescent magnetic nanoparticles [1], gold nanoparticles, upper conversion nanoparticles, etc. have been actively investigated for their applications in molecular imaging. Herein, we review main advances of UCNPs applied to tumor multimodality imaging [2] and simultaneous therapy [1] over the past few years, explore their application prospects, and discuss the concepts, issues, approaches and challenges, with the aim of improving the application of UCNPs in biomedical imaging and therapy in near future.

\section{Advances of preparation of UCNPs}

It is well known that fluorescence imaging is a key technique for biological studies and disease diagnosis due to its high temporal and spatial resolutions. Conventional fluorescence imaging process, namely down-conversion (DC), refers to emitting low-energy photon when excited by a high-energy light (always UV). It has some limitations such as causing DNA damage and cell death due to long-term irradiation with UV or other shortwavelength light, significant auto-fluorescence from 
biological tissues, low signal-to-noise ratio (SNR) and short penetration depth of short wavelength excitation light in biological tissues. The urgent needs of bioimaging technology, pharmaceutical engineering, diagnosis and treatment of diseases are challenging to find more efficient fluorescence probes than traditional organic dyes. Subsequently, semiconductor quantum dots (QDs) with tunable size-dependent emission, high quantum yields of photoluminescence (PL), broad excitation spectra and narrow emission bandwidths once evoked plenty of researchers' attentions. However, they also have intrinsic limitations such as inherent toxicity and chemical instability in the bio-system even incorporated with Föster resonance energy transfer (FRET) technology. In comparison with UV excitation, NIR excitation could minimize auto-fluorescence from biologicaltissues, penetrate into a larger depth, and is less harmful to cells as well $[3,4]$.

Especially, upconversion luminescence (UCL) is a process where low-energy light, usually NIR light (typically $980 \mathrm{~nm}$ ), is converted to visible light through the sequential absorption of multiple photons or energy transfers induced by a fairly low power $\left(1-10^{3} \mathrm{w} \mathrm{cm}^{-2}\right)$ continuous wave laser [5]. Up to date, as fluorescent biolabels, UCNPs have shown significant merits over the traditional organic fluorophores due to their attractive optical and chemical features such as low toxicity, large Stokes shifts, high resistance to photobleaching, and photochemical degradation. In particular, lanthanides (Ln)-doped UCNPs exhibit unique UCL properties, such as sharp emission lines (full width at half maximum $<12 \mathrm{~nm})$, long life times $(\sim \mathrm{ms})$, a large anti-Stokes shift, superior photostability, no blinking and no autofluorescence of biological samples $[4,6]$. All these make UCNPs an ideal choice as a new generation of luminescent probes for molecular imaging. In addition, UCNPs with a mesoporous or (and) hollow structure have been widely used as ideal carriers for drug delivery, gene therapy and photodynamic therapy due to their high specific surface and cavity volumes. With the progress of nanotechnology, a suite of typical approaches including thermal decomposition method, hydrothermal method, co-precipitation method and so on, have been developed to synthesize distinct UCNPs according to different requirements. These methods have been reviewed in previous review paper and introduced in Table 1 to make them more clear and concise.

Acting as versatile bioprobes applied in nanomedicine and nanotechnology, UCNPs should be provided with uniform size and morphology, excellent water-solubility, suitable surface functional groups for bioconjugation with various biomolecules as well as high luminescent efficiency. To meet these requirements, many efforts have been devoted to the controlled synthesis. For example, Zhao et al. [29] believed that it could precipitate phase transformation from $\alpha$ to $\beta$ just by tuning the hydrothermal temperature, time and the amount of $\mathrm{NaOH}$. More interesting, upon adjusting the amount of $\mathrm{NaOH}, \mathrm{NaF}$, $\mathrm{Re}^{3+}$ and ethanol, the morphologies for the $\beta-\mathrm{NaReF}_{4}$ can range from tubes, rods, wires, zigzagged rods to flowerpatterned disks. As the last element in the lanthanides, Lutetium cations have the smallest $\mathrm{Re}$ ionic radii, which may contribute to the exceptional performance of its fluorides [30]. To date, lutetium fluorides with different compositions, morphologies, and crystal phases have been successfully synthesized. However, owing to their size of micron level, most of them can't be implied in life systems. Recently, Li's group have resoundingly synthesized sub-20 nm [31] (Fig. 1) and sub-10 nm [32] water-stable Lu-UCNPs, which may be ideal building blocks for multimodal imaging(UCL/CT/MRI/PET) agents. Especially, the sub-20 nm NaLuF 4 co-doped $\mathrm{Yb}^{3+}$ and $\mathrm{Er}^{3+}\left(\mathrm{Tm}^{3+}\right)$ show about 10-fold stronger UCL emission than that of corresponding hexagonalNaYF ${ }_{4}^{-}$ based nanocrystals with a $20 \mathrm{~nm}$ diameter, forecasting $\mathrm{NaLuF}_{4}$ an ideal host for multimodal bioimaging probes.

In fact, hydrothermal treatment as a typical solution approach has been proven to be the most effective and convenient in the synthesis of orderly uniform, single crystalline and phase-pure UCNPs. However, hydrothermal and other analogous approaches often require an extended period, high temperature and pressure, in company with the exposure of many kinds of toxic byproducts, which may bring about environmental and

Table 1 Typical UC host materials and their synthetic strategies

\begin{tabular}{|l|l|l|}
\hline \multicolumn{1}{|c|}{ Methods } & \multicolumn{1}{|c|}{ Examples(Hosts) } \\
\hline $\begin{array}{l}\text { Hydrothermal/solvothermal } \\
\text { method }\end{array}$ & $\begin{array}{l}\mathrm{NaYF}_{4}[7], \mathrm{NaYbF}_{4}[8], \\
\mathrm{NaGdF}_{4}[9], \mathrm{LaF}_{3}[10]\end{array}$ & $\begin{array}{l}\text { Remarks } \\
\text { Specialized reaction vessels required. }\end{array}$ \\
\hline $\begin{array}{l}\text { Thermal decomposition } \\
\text { method }\end{array}$ & $\begin{array}{l}\mathrm{NaYF}_{4}[11], \mathrm{NaYbF}_{4}[12] \\
\mathrm{YaGdF}_{4}[13], \mathrm{LaF}_{3}[14],\end{array}$ & $\begin{array}{l}\text { High uniformity and monodisperse. } \\
\text { Rigorous experimental conditions Toxic precursors. }\end{array}$ \\
\hline Co-precipitation method & $\begin{array}{l}\mathrm{NaYF}_{4}[16], \mathrm{LaF}_{3}[17], \\
\mathrm{NaGdF}_{4}[18]\end{array}$ & Convenient. Post treatment usually required \\
\hline Sol-gel processing & $\mathrm{ZrO}_{2}[19]$ & Poor size control and considerable aggregation. \\
\hline Combustion synthesis & $\mathrm{Y}_{2} \mathrm{O}_{3}[20], \mathrm{Gd}_{2} \mathrm{O}_{3}[21]$ & Time and energy saving. Limited scope. \\
\hline Flame synthesis & $\mathrm{Y}_{2} \mathrm{O}_{3}[22]$ & Time saving. Limited scope. \\
\hline Two-phase synthesis & $\begin{array}{l}\mathrm{NaYF}_{4}[23], \mathrm{NaGdF}_{4}[24], \\
\mathrm{LaF}_{3}[25]\end{array}$ & Surface functional. \\
\hline Ionic liquid-based synthesis & $\mathrm{NaYF}_{4}[26]$ & $\begin{array}{l}\text { Environmental friendly. Recyclable raw material. } \\
\text { Poor size control. }\end{array}$ \\
\hline Microwave-assisted synthesis & $\mathrm{NaYF}_{4}[27], \mathrm{LaF}_{3}[28]$ & Time saving. Specialized reaction devices required. \\
\hline
\end{tabular}


http://nanobe.org

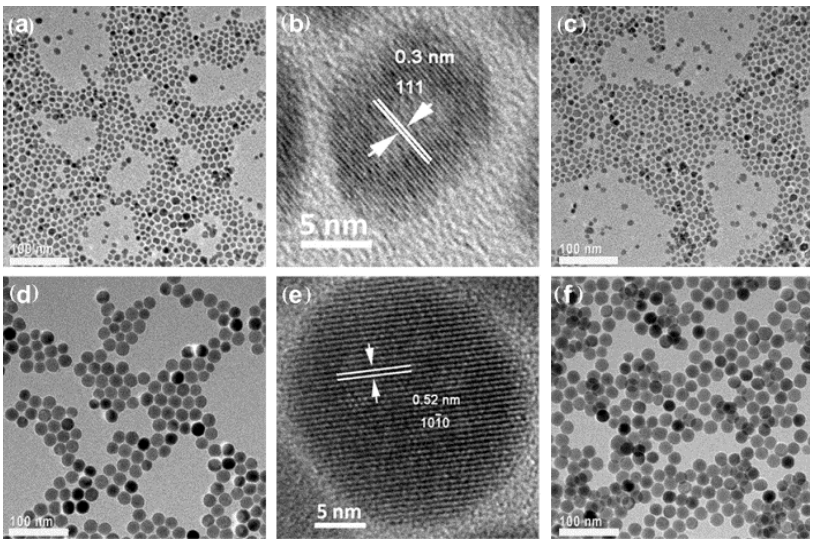

Fig. 1 TEM images of (a) NaLuF4:20\% Yb,2\%Er (OA-LuEr), (c) PAAcoated $\mathrm{NaLuF}_{4}: 20 \% \mathrm{Yb}, 2 \% \mathrm{Er}$ (PAA-LuEr), (d) $\mathrm{NaYF}_{4}: 20 \% \mathrm{Yb}, 2 \% \mathrm{Er}$ (OA-YEr), (f) PAA-coated $\mathrm{NaYF}_{4}: 20 \% \mathrm{Yb}, 2 \% \mathrm{Er}$ (PAA-YEr), HR-TEM of OA-LuEr (b) and OA-YEr(e). Reprinted with permission from Yang T, Sun Y, Liu Q, Li F. Cubic sub $20 \mathrm{~nm} \mathrm{NaLuF}_{4}$-based upconversion nanophosphors for high-contrast bioimaging in different animal species. Biomaterials 2012, 33:3733-3742 by ELSEVIER pulication.

safety problems. In addition, conventional synthetic strategies, namely two-step synthetic approaches, commonly need complicated surface modification to couple with functional groups for hydrophily and biocompatibility. Ionic liquids (ILs), owing to their unique properties such as negligible vapor pressure, wide electrochemical window, and thermal and chemical stability, have acted as a promising medium for the synthesis and have been regarded as a "green" alternative to the conventional organic solvents. Nevertheless, the asprepared products usually have poor shape-control, low dispersity and broad size-distribution [33]. Consequently, our group have adopted the notion of liquid-liquid twophase approach (the advantage of thermal decomposition method) and ILs as fluorine source (the advantage of IL-based strategy) and create a novel IL-OA dual phase method (Fig. 2), by which hydrophilic and hydrophobic Ln-doped $\mathrm{NaYF}_{4}, \mathrm{NaGdF}_{4}, \mathrm{NaYbF}_{4}, \mathrm{LaF}_{3}$ could be selectively synthesized in a one-pot step [33-37]. We are confident that other kinds of UCNPs with excellent morphology and UC emissions can be gained by this method as well. To sum up, one-step synthesis can simplify the reaction procesure, while products by twostep synthesis have better uniformity and monodispersity.

\section{Advances of UCNPs for molecular imaging}

In addition to their own unique properties, UCNPs and their nanocomposites have also attracted enormous attentions in that the integration systems often exert a more superior performance in a sense. "Nanocomposite polymers" are polymers that have dispersed phase with ultrafine dimension, typically on then a nanometer scale. The introduction of inorganic nanoparticles into a polymer matrix has proved to be an effective method to improve the performance of polymer materials and bring about novel properties in them. Up to date, the in situ photopolymerization method has been applied to synthesize bulk nanocomposites consisting of hydrophobic $\mathrm{NaYF}_{4}: \mathrm{Yb}^{3+}, \mathrm{Er}^{3+}\left(\mathrm{Tm}^{3+}\right) \mathrm{NPs}$ as the filler and poly(methyl methacrylate) (PMMA) as the host material by Lin and co-workers [38]. The nanocomposites were transparent in visible spectral region and exhibited strong green and blue UC luminescence upon $980 \mathrm{~nm}$ laser excitation. A multi-functional nanoprobe of polyethylene glycol (PEG) modified $\mathrm{BaGdF}_{5}: \mathrm{Yb}$, Er UCNPs for trimodal bioimaging of fluorescence, CT and MRI are demonstrated the first time by Hao and co-workers [39] (Fig. 3). Zhang et al. [40] have synthesized pure cubic $\mathrm{PVP} / \mathrm{NaYF}_{4}$ nanocrystals co-doped with $\mathrm{Re}^{3+}$ such as $\mathrm{Yb}, \operatorname{Er}(\mathrm{Tm})$ using PVP as a chelating agent and stabilizer. Moreover, a uniform layer of silica with the thickness adjustable down to $1-3 \mathrm{~nm}$ is coated onto the PVP/NaYF nanocrystals to form a core-shell structure.

As we know, gold nanomaterials have been utilized for optical imaging, either via two-photon fluorescence or via surface plasmon resonance (SPR) scattering. However, the real-time visualization and remote detection cannot be achieved at the same time because of lacking an appropriate light source [41]. Yan et al. [42] first

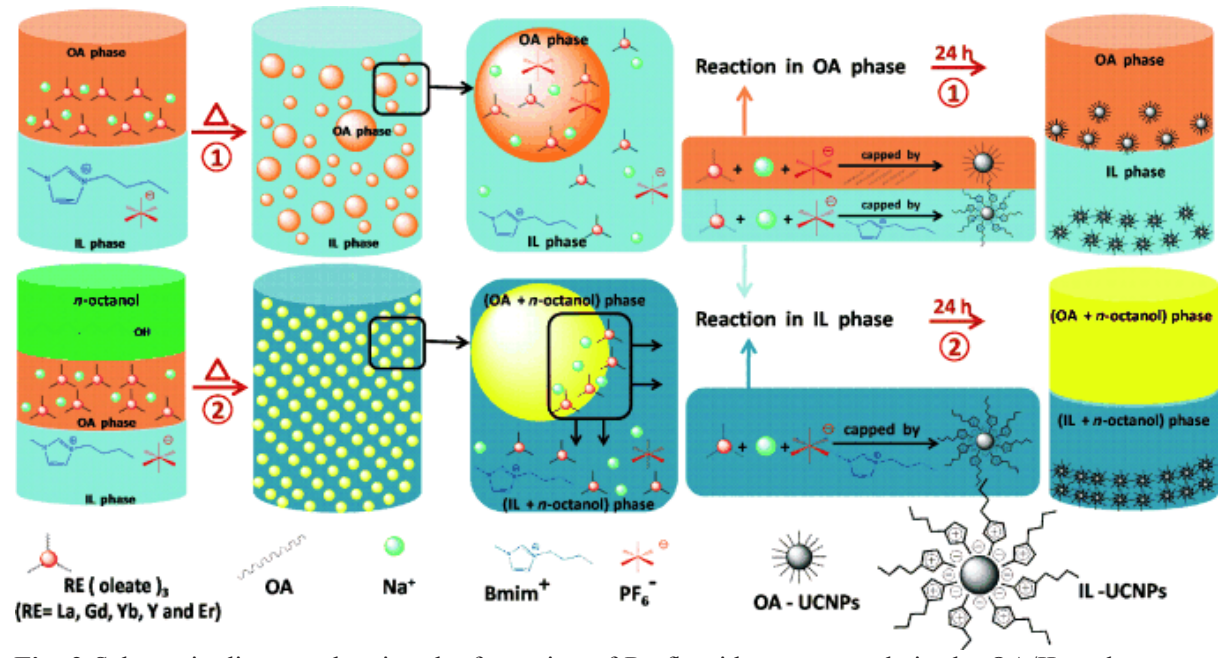

Fig. 2 Schematic diagram showing the formation of Re fluoride nanocrystals in the OA/IL and n-octanolinduced two-phase systems. Reprinted with permission from He M, Huang P, Zhang C, Ma J, He R, Cui D. Phase- and size-controllable synthesis of hexagonal upconversion rare-earth fluoride nanocrystals through an oleic acid/ionic liquid two-phase system. Chemistry 2012, 18:5954-5969 by WILEY publication. 
http://nanobe.org
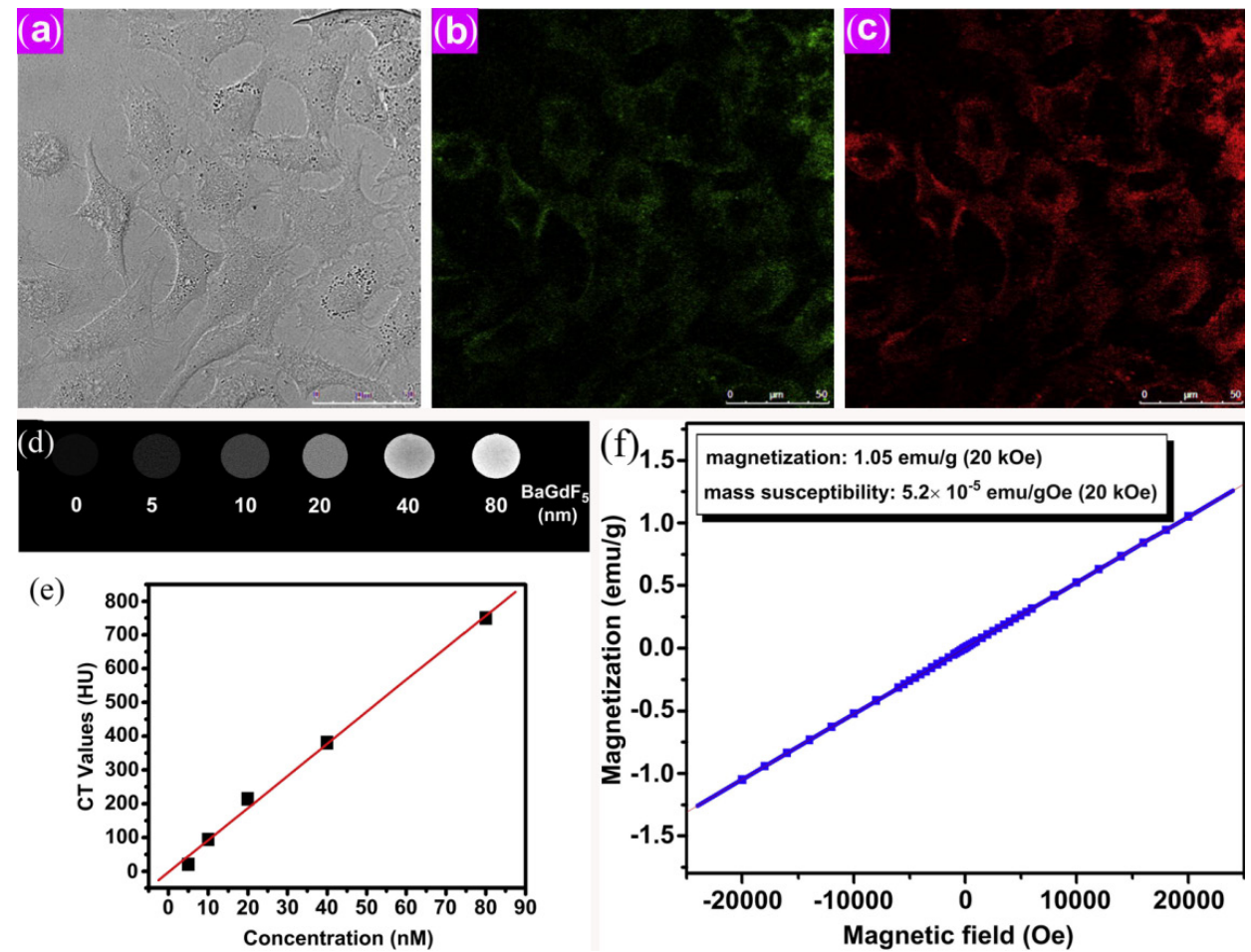

(e)

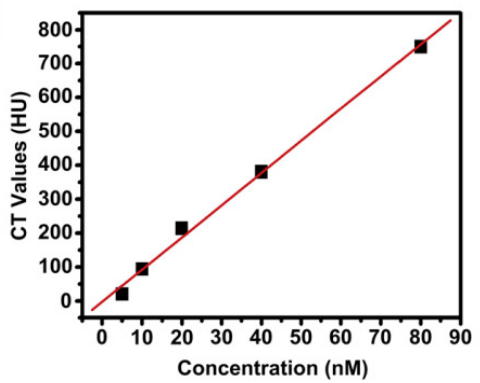

Fig. 3 (a-c) In vitro bioimaging of the PEG-modified BaGdF $5: Y b / E r$ colloidal UCNPs in HeLa cells.(d) CT images of water solutions under different concentrations of PEG-modified $\mathrm{BaGdF}_{5}: \mathrm{Yb} / \mathrm{Er} \mathrm{UCNPs}$, (e) The measured $\mathrm{CT}$ values (Hounsfield units, $\mathrm{HU}$ ) of PEG-modified $\mathrm{BaGdF}_{5}: \mathrm{Yb} / \mathrm{Er}$ UCNPs. (f) Magnetization as a function of applied field for the PEG-modified $\mathrm{BaGdF}_{5}: \mathrm{Yb} / \mathrm{Er} \mathrm{UCNPs}$ at room temperature. Reprinted with permission from Zeng S, Tsang MK, Chan CF, Wong KL, Hao J. PEG modified BaGdF (5):Yb/Er nanoprobes for multi-modal upconversion fluorescent in vivo X-ray computed tomography and biomagnetic imaging. Biomaterials 2012, 33:9232-9238 by ELSEVIER publication.

observed the enhanced UCLemission of $\beta-\mathrm{NaYF}_{4}: \mathrm{Yb}$,Er nanocrystals when coupled to Ag nanowires via a direct assembly method. The enhancement factor for the red emission $(650 \mathrm{~nm})$ was larger than that for green emission $(550 \mathrm{~nm})$, and could be further increased by the use of $\mathrm{Ag}$ islands composed of larger $\mathrm{Ag}$ particles. Song, et al. [41] have presented the synthesis of core-shell structured $\beta-\mathrm{NaYF}_{4}: \mathrm{Yb}^{3+}, \mathrm{Er}^{3+} @ \mathrm{AgNPs}$ and their unique bio-functional properties. Consequently the core/shell NPs were expected to be atempting therapeutic agent for tumor ablation with bioimaging and thermal treatment in real time. Qin et al. [43] have explained that it was the plasmon field enhancement effect that was the main reason for the improved UC emission efficiency. In addition, unselectively enhanced multicolour UC emissions and low pumping threshold were achieved in
$\mathrm{Au} @ \beta-\mathrm{NaYF}_{4}: \mathrm{Yb}, \mathrm{Tm}$ hybrid nanostructures. Zhang et al. [44] reported a poly-(aminoacid)-templated gold-shell encapsulation of the silica coatedNaYF $: \mathrm{Yb}$,Er UCNPs, showing how a deft tuning of SPR peak from visible to NIR region dramatically transformed the luminescence quenching into an enhancement effect and how theNPs were used for combined UC fluorescence and dark field light scattering imaging. At one time, most commercially available two-photon fluorophoresare organic dyes that exhibit relatively low two-photon absorption crosssections, fluorescence quantum yield and photochemicalinstability (photobleaching). Many efforts have been made to prepare better organic fluorophores with customized properties, one of which is UCNPs. However, the practical applicability of most efficient known UC materialsat moderate light intensities is limited by their
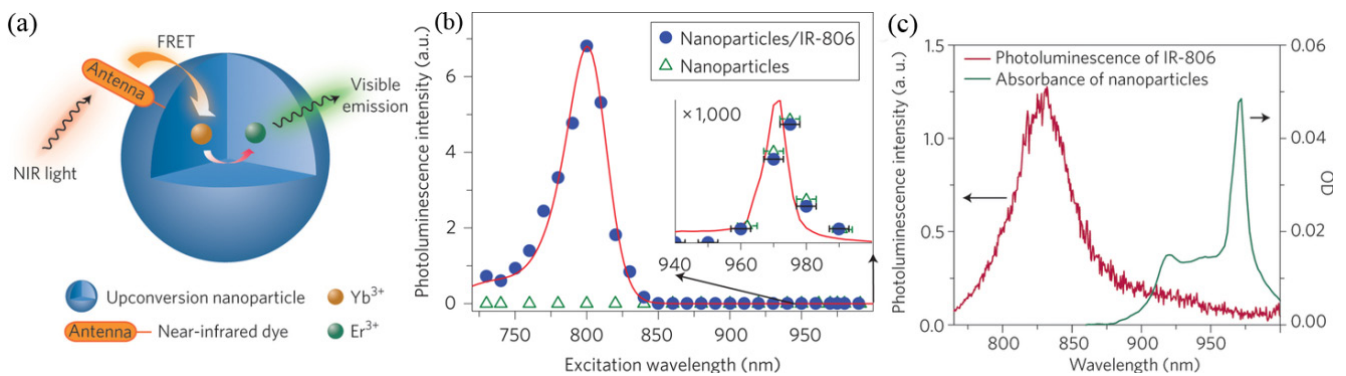

Fig. 4 (a) Schematic of the dye-sensitized nanoparticle. (b) Experimental upconversion excitation spectra of

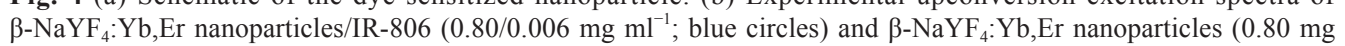
$\mathrm{ml}_{-1}$; green triangles), both dissolved in $\mathrm{CHCl}_{3}$. (c) Emission (red) and absorption (green) spectra of the cyanine dye (IR-806) and the $\mathrm{NaYF}_{4}: \mathrm{Yb}$,Er nanoparticles. Reprinted with permission from Xie X, Liu X. Photonics: Upconversion goes broadband. Nat Mater 2012, 11:842-843 by NATURE publication. 
relatively weak and narrowband near-infrared absorption. Lately, Hummelen et al. [45] introduced an analogy that an organic near-infrared dye is used as an antenna for

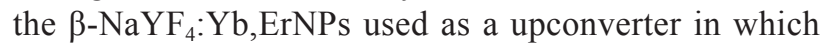
dyes were endowed with a new role in UC. The overall UC process is dramatically enhanced (by a factor of $\sim 3,300$ ) as a result of increased absorption (Fig. 4b). The conclusion has also been confirmed by Liu and coworkers [46] (Fig. 4a and Fig. 4c).

\subsection{Advances of UCNPs for Cell Tracking, Labeling and Imaging}

Zhang et al. [47] were able to track subtle changes in the distribution of the transplanted cells at high resolution in real time within a localized field of view by using a high magnification $40 \times$ water-immersion objective. Interestingly, Liu's group synthesized a series of UCNPs with different emission colors and used them for multicolor cancer cell labeling, realizing in vivo cell tracking by UCL imaging [48]. Then, they regarded UCNPs as an exogenous contrast agent, highlighting the promise of using UCNPs as a new type of ultra-sensitive probes for labeling and in vivo tracking of stem cells at nearly the single cell level [49]. Recently, Li's group [50] first achieved available quantification imaging by endowing ${ }^{153} \mathrm{Sm}$-postlabeling UCNP bifunction of radioactive property and UCL (Fig. 5a). This isotope labeling method showed rapid treatment time of $<1 \mathrm{~min}$, high labeling yield of $>99 \%$, and without usage of organic solvents, and was also suitable for most Redoped NPs to track their in vivo behaviors (Fig. 5b). Lately, our group [35] prepared folic acid (FA)-conjugated silica-modified $\mathrm{LaF}_{3}: \mathrm{Yb}, \mathrm{Tm}$ UCNPs (UCNPs@ $\mathrm{SiO}_{2}-\mathrm{FA}$ ) with high La content in a single particle. The cell imagine turned out that only very weak background fluorescence is observed, suggesting the as-prepared samples have a perfect SNR. Very recently, our group synthesized Ln-doped onedimensional NaGdF4submicrorods $(\sim 100 \mathrm{~nm}$ in diameter, 200-300 nm in length) and porous NaYF4microrods ( $\sim 1 \mu \mathrm{m}$ in diameter, 3-5 $\mu \mathrm{m}$ in length) by a facile ethylenediaminetetraacetic acid (EDTA)-assisted hydrothermal method, and both have good biocompati-bility and strong CT signal intensity [51].

Molecular imaging techniques, such as CT, MRI, PET as well as UCL have played very important roles in biomedical research and disease diagnosis. However, information supplied from single modal imaging can't satisfy the higher requirements on the efficiency and accuracy for clinical diagnosis and medical research any more, due to its limitation and default rooted in single modal imaging technique itself [52]. To date, Re-doped NPs have been applied successfully as probes for dualmodality or trimodality imaging including CT, PET and MRI. Due to the super-paramagnetic of iron oxide, iron oxide nanoparticles (IONPs) combined with UCNPs nanocomposites have been widely implied in multimodality imaging especially in $\mathrm{T}_{2}$-enhanced MR imaging. Multifunctional nanoparticles (MFNPs) based on UCNPs with combined optical and magnetic properties are synthesized via a simple layer-by-layer self-assembly strategy by Liu's group [53]. PEGylated MFNPs can be used for targeted UCL, MR, and dark-field imaging; for molecular and magnetic targeted photothermal therapy (PTT) of cancer cells; and as a contrast agent for in vivo dual-modal UCL/MR imaging in mice. Besides, IONPs serve as a "buffer" layer between the luminescent UCNP core and the outside gold shell to reduce the UCL quenching effect. However, it is proven that the intensity of UCL emission will be weaker in the presence of the $\mathrm{Fe}_{3} \mathrm{O}_{4}$ shielding, because both excitation and emission light are absorbed by the $\mathrm{Fe}_{3} \mathrm{O}_{4}$ shell. Therefore, a different core-shell nanostructure with $\mathrm{Fe}_{3} \mathrm{O}_{4}$ NPs as core and Re-UCNPs as shell has been realized by Li's group [54], showing excellent UCL, MR and CT properties. One shortcoming of this nanoparticle is its relatively large size $(\sim 330 \mathrm{~nm}$ in diameter) because of the presence of large $\mathrm{Fe}_{3} \mathrm{O}_{4}$ nanoparticle core. Further reduction in the size of this core-shell nanoparticle is still in progress.

On the other hand, it has been figured out that $\mathrm{Gd}^{3+}$ is an another good paramagnetic source when doped with Reions as a host (typically $\mathrm{NaGdF}_{4}$ ) in $\mathrm{T}_{1}$-enhanced $\mathrm{MR}$

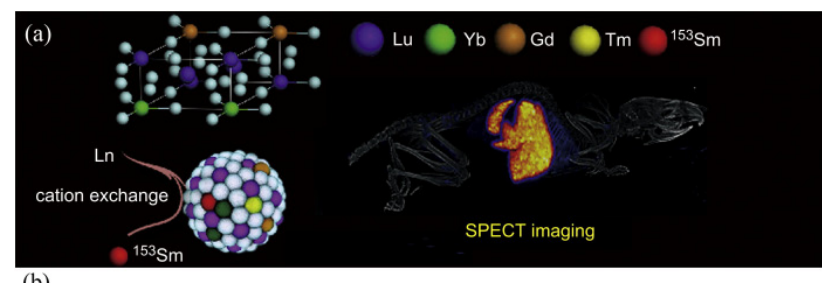

(b)

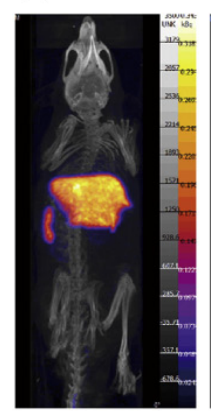

$60 \min$
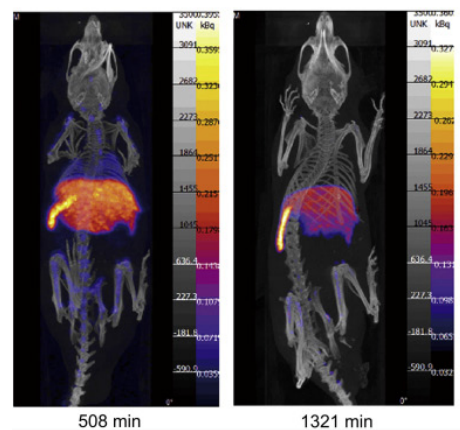

$1321 \mathrm{~min}$

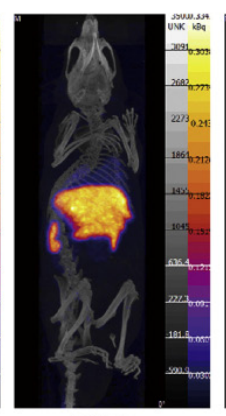

$208 \min$

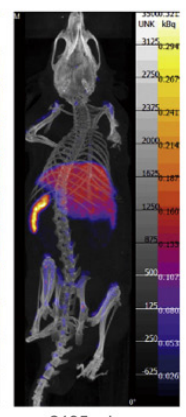

$2195 \mathrm{~min}$

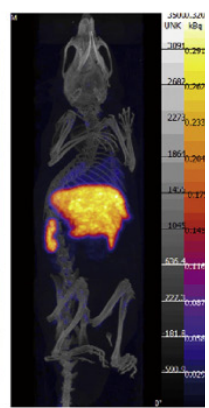

$238 \mathrm{~min}$

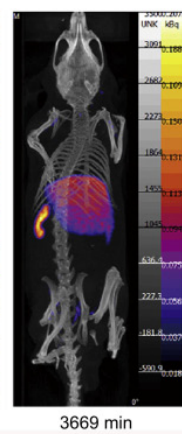

Fig. 5 (a) Schematic representation of $153 \mathrm{Sm}$-postlabeled Cit-UCNP by cation exchange for in vivo quantitative tracking. (b) In vivo SPECT/CT imaging by nano SPECT/CT plus at different time point $(60,123,208$, 238, 508, 1321, 2195 and $3669 \mathrm{~min}$ ) after intravenous injection of Cit153Sm-UCNP. Reprinted with permission from Sun Y, Liu Q, Peng J, Feng W, Zhang Y, Yang P, Li F. Radioisotope post-labeling upconversion nanophosphors for in vivo quantitative tracking. Biomaterials 2013, 34:2289-2295 by ELSEVIER publication. 
imaging. Muhammad et al. [55] created a seedmediated synthetic route to prepare core-shell structured $\mathrm{NaYF}_{4}: \mathrm{Yb}$, $\mathrm{Er} / \mathrm{NaGdF}_{4}$ nanocomposites. They believed that the epitaxial growth of a gadolinium layer on an UC lanthanide seed not only improved its upconversion fluorescence, but also produced a paramagnetic shell with high magnetic resonance relaxivity. Song and co-workers [56] synthesized $\mathrm{NaGdF}_{4}: \mathrm{Yb}^{3+}, \mathrm{Er}^{3+}$ and $\mathrm{NaGdF}_{4}: \mathrm{Yb}^{3+}$, $\mathrm{Tm}^{3+}$ UCNPs by using new combination of lanthanide chloride and sodium TFA as the precursors. To further evaluate the magnetic properties of $\mathrm{NaGdF}_{4}$ : $\mathrm{Yb}^{3+}, \mathrm{Er}^{3+}$, the aqueous solution of UCNPs at various concentrations was measured for their $T_{1}$ relaxation time. MRI showed the $\mathrm{Gd}^{3+}$ concentration dependent contrast effect of the UCNPs, and UCNPs with smaller size of $10 \mathrm{~nm}$ exhibited higher $\mathrm{T} 1$ contrast enhancing effect. It should be noted that UCNPs doped with $\mathrm{Gd}^{3+}$ can also show up excellent paramagnetic properties at room temperature, proven by Hao and co-workers [57]. Owing to the large magnetic moment of $\mathrm{Gd}^{3+}$, the $\mathrm{Gd}^{3+}$-doped $\mathrm{NaLuF}_{4}$ nanocrystals could be used as a MRI agent aswell. Our group [51] have done some quantificational research about the paramagnetism behavior of $\mathrm{Yb}^{3+}$ and $\mathrm{Ln}^{3+}$ doped 1-dimensionalNaYF 4 and $\mathrm{NaGdF}_{4}$ microcrystals. It turned out that both of them can exhibit paramagnetism at 300 $\mathrm{K}$ whereas $\mathrm{NaYF}_{4}$ microcrystals present a near superparamagnetic behavior at the magnetic range of -2 to 2 kOe. Both UCNPs exhibit superparamagnetic behavior at $2 \mathrm{~K}$. Their saturation magnetizations were 12.1 and 129.2 emu g ${ }^{-1}$ for $\mathrm{NaYF}_{4}$ and $\mathrm{NaGdF}_{4}$ crystals, respectively.

\subsection{Advances of UCNPs for drug delivery and simultaneous therapy}

UCNPs are also interesting nanocarriers for gene or drug delivery [58-61]. Especially, Liu's group have made a great effort and paved the way for multi-functional therapy in this field. For example, PEGylated UCNPs were loaded with a commonly used chemotherapy molecule; doxorubicin (DOX). The loading and releasing of DOX from UCNPs were controlled by varying $\mathrm{pH}$, with an increased drug dissociation rate in acidic environment, favorable for controlled drug release. It was found that DOX was shuttled into cells by the UCNP nano carrier and released inside cells after endocytosis. Besides DOX, this non-covalent drug loading strategy can also be used for loading of photosensitizer molecules on UCNPs for potential NIR light induced photodynamic therapy (PDT) [58], whose conclusion is similar to Zhao's [60]. Liu's group further encapsulated UCNPs together with IONPs, obtaining an UC-IO@polymer multi-functional nanocomposite system via a microemulsion method. Then, DOX was also loaded into the nanocomposite, forming an UC-IO@polymer-DOX complex, which enabled novel imaging-guided and magnetic targeted drug delivery [59] (Fig. 6). Recently, Lin and co-workers have achieved NIR-light-activated photopolymerization and controlled drug delivery for PEGDA hydrogels by utilizing multicolor UCL of Re-doped UCNPs. The loading capacity of zinc(II) phthalocyanine could reach $12.3 \%$ into UCNPs-PEGDA hybrid microspheres [62].

Additionally, mesoporous structures are one kind of good drug delivery tools. LaF3: $\mathrm{Yb}^{3+}, \mathrm{Er}^{3+} @ \mathrm{nSiO}_{2} @ \mathrm{mSiO}_{2}$ NPs were synthesized by Zhang and co-workers. Owning to the big enough surface area and pore volume of outer mesoporous silica shell, Ibuprofen (IBU) could be stored in the channels of the microspheres, and most of the drug molecules incorporated could be released to the simulated body fluid (SBF) in $70 \mathrm{~h}$ [63]. Very recently, Lin and coworkers [61] novelly synthesized $\alpha-\mathrm{NaYF}_{4}: \mathrm{Yb}^{3+}, \mathrm{Er}^{3+}$ nanospheres $(\sim 130 \mathrm{~nm})$ with mesoporous shell and hollow interior structure (HMNPs) tin which anti-cancer drug carriers could be leaded. Then, the drug storage/release

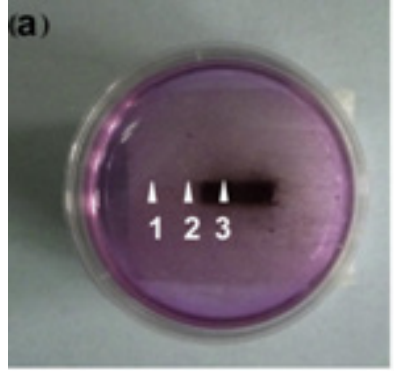

(b)

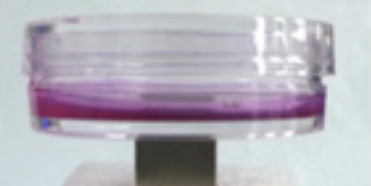

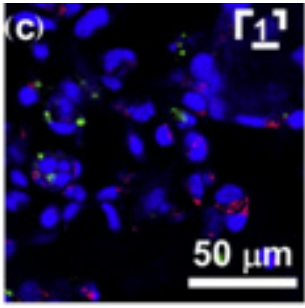

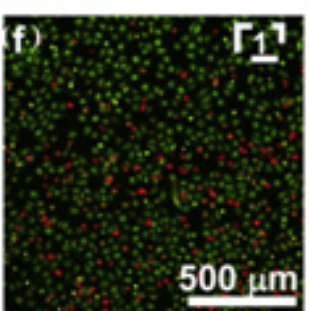

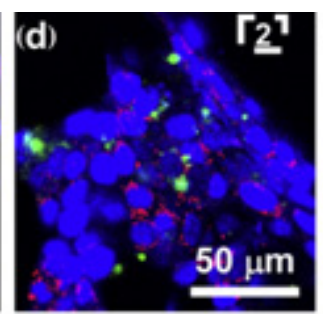
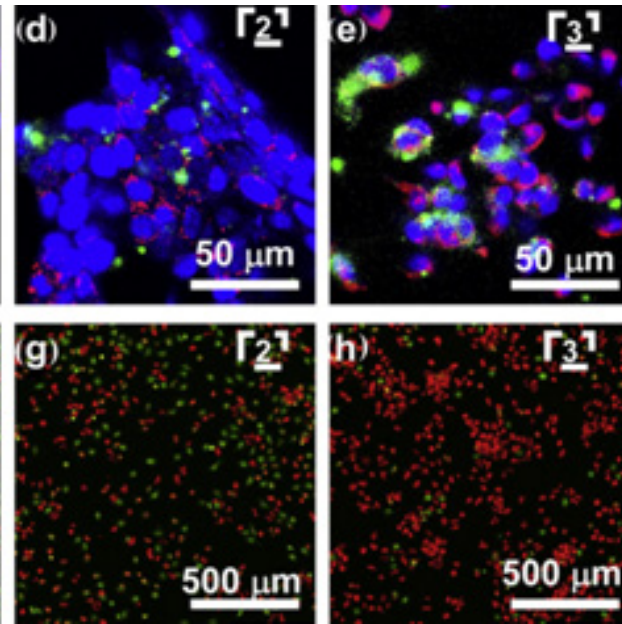

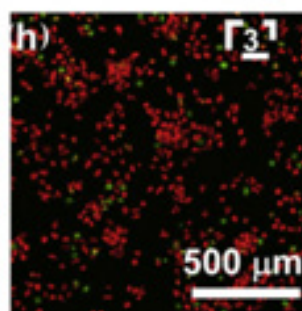

Fig. 6 (a\&b)Magnetic targeted drug delivery. (c-e) Photos of the cell culture dish with a magnet placed under the center of the dish. (f-h) Confocal UCL and FL images of HeLa cells after being incubated with UC-IO@Polymer-DOX in the presence of a magnetic field. Confocal FL images of calcein AM (green, live cells) and PI (red, dead cells) co-stained cells after magnetic targeted drug delivery. The above images were taken at three different locations in the culture dish: (1) Far from the magnet (c\&f); (2) Close to the magnet (d\&g); (3) Right above the magnet (e\&h). Reprinted with permission from Xu H, Cheng L, Wang C, Ma X, Li Y, Liu Z. Polymer encapsulated upconversion nanoparticle/iron oxide nanocomposites for multimodal imaging and magnetic targeted drug delivery. Biomaterials 2011, 32:9364-9373 by ELSEVIER publication. 
properties were tested and it turned out that FA-modified HMNPs exhibited greater cytotoxicity than DOX-loaded $\alpha-\mathrm{NaYF}_{4} \mathrm{NPs}$, indicating tremendous potential for simultaneous targeted anti-cancer drug delivery and cell imaging, agreeing with Zhao and co-workers [64].

The siRNA and DNA have been attached to UCNPs respectively. According to Ye's report [3], siRNA was attached to anti-Her2 antibody conjugated $\mathrm{NaYF}_{4}: \mathrm{Yb}^{3+}$, $\mathrm{Er}^{3+} @ \mathrm{SiO}_{2}$ and the delivery of these NPs to SK-BR-3 cells was studied, having achieved targeted delivery of siRNA to cancer cells. Lu and co-workers [65] introduced an exceptionally simple strategy to prepare uniform DNA modified UCNPs as versatile bioprobes. Furthermore, DNA molecules on the DNA-UCNPs retained their biorecognition ability, allowing programmable assembly of hybrid nanostructures. More importantly, these DNAUCNPs were capable of crossing cell membranes without the need of transfection agents, and their use as agents for bioimaging and DNA delivery were also demonstrated. Very recently, Xing and co-workers reported that upon NIR light irradiation, the photocaged linker on the SiUCNPs surface could be cleaved by the upconverted UV light and thus initiated the intracellular release of the siRNA. The in vitro agarose gel electrophoresis and intracellular imaging results indicated that the Si-UCNPsbased gene carrier system allowed effective siRNA delivery and the applications of NIR light instead of direct high energy UV irradiation may greatly guarantee less cell damage [66].

Except for drug loading and delivery, UCNPs have been extensively applied to targeted therapy of disease, including chemotherapy, PDT, PTT. Recently, Zhang's group [67] have creatively synthesized a meso-porous multifunctional UC luminescent and magnetic "nanorattle" nanomaterial that can be directed by an external magnetic field to a specific target with a therapeutic agent in the mesoporouses, meanwhile having low cytotoxicity and excellent cell imaging properties. PDT, which is known as a non-invasive medical method to treat diseases such as cancer [68], utilizes a photosensitizer working as a light-sensitive drug to treat the target tissue locally upon the irradiation of light with appropriate wavelengths. Conventional PDT is limited by the penetration depth of visible light needed for its activation; however, UCNPs have shown tremendous potential in PDT [69-73]. As early as in 2007, UCNPs were first introduced into PDT by Zhang and co-workers as a new type of photosensitizers and brought close to the target cancer cells through antigen-antibody interaction with good specificity and versatility [69]. In 2008, PEI/ $\mathrm{NaYF}_{4}: \mathrm{Yb}^{3+}, \mathrm{Er}^{3+} \mathrm{NPs}$ with green/red emission, playing a role of nanotransducers to enable PDT in deep tissues were targeted to folate receptors on human colon cancer cells by Zhang's group [70]. After that, they incorporated photosensitizers into the porous silica shell of UCNPs for PDT of cancer cells [71], and then further developed a means to amplify the therapeutic function of UC fluorescence-based PDT [72] by bringing a combination of two photosensitizers (as opposed to previous singlephotosensitizer approaches) to a treatment site without the need for complicated multiple-wavelength excitation (Fig. 7). Recently, dual-modal in vivo tumor imaging and PDT were performed using photosensitizer-conjugated $\beta-\mathrm{NaYF}_{4}: \mathrm{Yb}, \mathrm{Er} / \mathrm{NaGdF}_{4}$ core-shell UCNPs by Hyeon and co-workers [73].

PTT is a treatment regime involving irradiation of diseased tissue with electromagnetic radiation to cause thermal damage. The spatial specificity and minimalinvasiveness make PTT an attractive therapeutic model, as compared to surgery or other invasive therapeutic procedures [68]. Chou and co-workers [74] synthetized a UCNP/silica/Au structure that showed green emission coupled with the surface plasmon of Au, which leaded to rapid heat conversion. The thermal damage was efficient enough to destroy BE (2)-C cancer cells and showed strong potential in PTT. On the other hand, HepG2 cells from human hepatic cancer and Bcap-37 cells from human breast cancer incubated with the $\mathrm{NaYF}_{4}: \mathrm{Yb}^{3+}$, $\mathrm{Er}^{3+} @ \mathrm{Ag}$ nanocomposites were found to undergo photothermally induced death on exposure to $980 \mathrm{~nm}$ NIR light by Song and co-workers [75], and the optimum mortality approaches $95 \%$ with a power density of 1.5 (a)

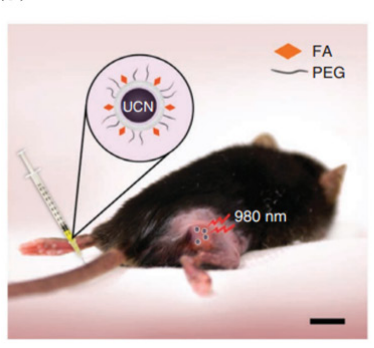

(b)

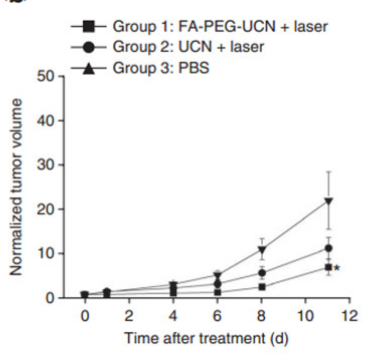

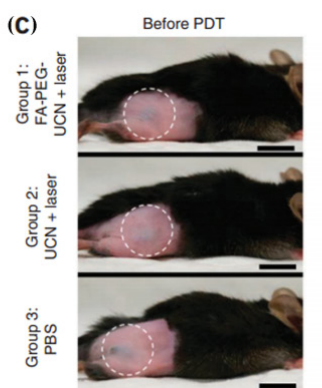

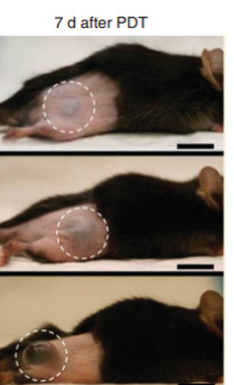

Fig. 7 Targeted in vivo PDT of a subcutaneous tumor model injected with FA-PEG-UCNs. (a) Schematic diagram showing UCN-based targeted PDT in a mouse model of melanoma intravenously injected with UCNs surface modified with folic acid (FA) and PEG moieties. Scale bar, $10 \mathrm{~mm}$. (b) Change in tumor size as a function of time after treatment to assess the effectiveness of UCN-based mediated targeted PDT in tumor-bearing mice intravenously injected with FA-PEG-UCNs. (c) Representative gross photos of a mouse from each group 1-3 intravenously injected with FA-PEGUCNs, unmodified UCNs or PBS showing the change in tumor size (highlighted by dashed white circles) before $(0 \mathrm{~d})$ and $7 \mathrm{~d}$ after PDT treatment. Scale bars, $10 \mathrm{~mm}$. Reprinted with permission from Idris NM, Gnanasammandhan MK, Zhang J, Ho PC, Mahendran R, Zhang Y. In vivo photodynamic therapy using upconversion nanoparticles as remotecontrolled nanotransducers. Nat. Med. 2012, 18:1580-1585 by NATURE AMERICA publication. 
$\mathrm{W} \mathrm{cm}{ }^{-2}$, which is much lower than that reported for $\mathrm{Au}$ nanoshells and nanorods. Liu et al. [76,77] have further presented that multifunctional UCNPs can be used for in vitro targeted UCL, MR, dark-field imaging and magnetic targeted PTT of cancer cells, highlighting the promise of using UCNPs for cancer theranostics.

\section{Conclusion and outlook}

UCNPs have aroused wide attentions in recent years, and are actively investigated for their application in molecular imaging. However, to our best knowledge, it does exist a number of challenges and hardship to overcome in the field, which may lead the research trend in the near future in turn. For example, conventional synthetic strategies always need post modification to couple with functional groups for hydrophily and biocompatibility. However, one-pot methods for synthetizing hydrophilic UCNPs are still in the infant, as well as the problem on how to improve their monodispersity. As is well-known, UCNPs in hexagonal phase has stronger luminescence efficiency than that in cubic phase while the mechanism hasn't been demonstrated systematically yet and more investigations are still needed. On the other hand, despite UCNPs have exhibited superior performance compared with QDs, their luminescent intensity and color diversity always can't catch up with the latter. Thus, how to improve UCL intensity tactfully is extremely urgent. We believe that new generation of UCNPs with strong fluorescent signals will be fabricated and own great potential in applications such as molecular imaging and simultaneous therapy in near future.

\section{References}

1 Cui D.X., Han Y, Li Z, Song H, Magnetic nanoprobes for in vivo targeted imaging and hyperthermia therapy of prostate cancer. Nano Biomed. Eng. 2009,1:61-64.

2 Bao C.C., Yang H, Sheng P, Song H, Cui D. Cloning, expression, monoclonal antibody preparation of human gene nbeall and its application in targeted imaging of mouse glioma. Nano Biomed. Eng. 2009,1:50-56.

3 Shan J., et al., NIR-to-visible upconversion nanoparticles for fluorescent labeling and targeted delivery of siRNA. Nanotechnology 2009. 20(15): 155101.

4 Zhou J., Liu Z., Li F., Upconversion nanophosphors for small-animal imaging. Chem. Soc. Rev. 2012. 41(3): 1323-49.

5 Xiong L.Q., et al., Synthesis, characterization, and in vivo targeted imaging of amine-functionalized rare-earth up-converting nanophosphors. Biomaterials 2009. 30(29):5592-600.

6 Zhou J., et al., Dual-modality in vivo imaging using rare-earth nanocrystals with near-infrared to near-infrared (NIR-to-NIR) upconversion luminescence and magnetic resonance properties. Biomaterials 2010. 31(12): 3287-3295.

7 Zhang F., et al., Uniform Nanostructured Arrays of Sodium RareEarth Fluorides for Highly Efficient Multicolor Upconversion Luminescence. Angew. Chem. Int. Ed. 2007. 46(42): 7976-7979.

$8 \mathrm{Li}$, C. et al., Shape controllable synthesis and upconversion properties of $\mathrm{NaYbF}_{4} / \mathrm{NaYbF}_{4}: \mathrm{Er}^{3+}$ and $\mathrm{YbF}_{3} / \mathrm{YbF}_{3}: \mathrm{Er}^{3+}$ microstructures. J. Mater. Chem. 2008. 18(12): 1353-1361.

9 Feng W., et al., Multicolour PEI/NaGdF $4: \mathrm{Ce}^{3+}, \mathrm{Ln}^{3+}$ nanocrystals by single-wavelength excitation. Nanotechnology 2007. 18(2): 025701.

10 Liu C. Chen D., Controlled synthesis of hexagon shaped lanthanidedoped $\mathrm{LaF}_{3}$ nanoplates with multicolor upconversion fluorescence. J. Mater. Chem. 2007. 17(37): 3875-3880.

11 Mai H.X., et al., High-Quality Sodium Rare-Earth Fluoride
Nanocrystals: Controlled Synthesis and Optical Properties. J. Am. Chem. Soc. 2006. 128(19): 6426-6436.

12 Ehlert O., et al., A Four-Color Colloidal Multiplexing Nanoparticle System. ACS Nano, 2008. 2(1): 120-124.

13 Wang F., et al., Simultaneous phase and size control of upconversion nanocrystals through lanthanide doping. Nature, 2010. 463(7284) : 1061-5.

14 Zhang Y.W., et al., Single-Crystalline and Monodisperse $\mathrm{LaF}_{3}$ Triangular Nanoplates from a Single-Source Precursor. J. Am. Chem. Soc. 2005. 127(10): 3260-3261.

15 Das G.K., Tan T.T.Y. Rare-Earth-Doped and Codoped $\mathrm{Y}_{2} \mathrm{O}_{3}$ Nanomaterials as Potential Bioimaging Probes. J. Phys. Chem. C 2008. 112(30): 11211-11217.

16 Heer S., et al., Highly Efficient Multicolour Upconversion Emission in Transparent Colloids of Lanthanide-Doped $\mathrm{NaYF}_{4}$ Nanocrystals. Adv. Mater. 2004. 16(23-24): 2102-2105.

17 Sivakumar S., Diamente P.R., van Veggel F.C.J.M. Silica-Coated $\mathrm{Ln}^{3+}$-Doped $\mathrm{LaF}_{3}$ Nanoparticles as Robust Down- and Upconverting Biolabels. Chem. A Eur. J. 2006. 12(22): 5878-5884.

18 Aebischer A., et al., Visible light emission upon near-infrared excitation in a transparent solution of nanocrystalline $\beta-\mathrm{NaGdF}_{4}$ : $\mathrm{Yb}^{3+}, \mathrm{Er}^{3+}$. Chem. Phys. Lett. 2005. 407(1-3): 124-128.

19 Patra A., et al., Upconversion in $\mathrm{Er}^{3+}: \mathrm{ZrO}_{2}$ Nanocrystals. J Phys. Chem. B 2002. 106(8):1909-1912.

20 Shuang Fang L., et al., Upconverting nanophosphors for bioimaging. Nanotechnology 2009. 20(40): 405701.

$21 \mathrm{Xu} \mathrm{L}$., et al., Synthesis and upconversion properties of monoclinic $\mathrm{Gd}_{2} \mathrm{O}_{3}: \mathrm{Er}^{3+}$ nanocrystals. Optic. Mater. 2008. 30(8): 1284-1288.

22 Kong W., Shan J., Ju Y. Flame synthesis and effects of host materials on $\mathrm{Yb}^{3+} / \mathrm{Er}^{3+}$ co-doped upconversion nanophosphors. Mater. Lett. 2010.64(6): 688-691.

23 Liu C., et al., Monodisperse, size-tunable and highly efficient [small beta]- $\mathrm{NaYF}_{4}: \mathrm{Yb}, \mathrm{Er}(\mathrm{Tm})$ up-conversion luminescent nanospheres: controllable synthesis and their surface modifications. J. Mater. Chem. 2009. 19(21): 3546-3553.

24 Liu C., et al., Morphology- and phase-controlled synthesis of monodisperse lanthanide-doped $\mathrm{NaGdF}_{4}$ nanocrystals with multicolor photoluminescence. J. Mater. Chem. 2009. 19(4): 489-496.

25 Liu C., et al., Size and morphology controllable synthesis of oildispersible $\mathrm{LaF}_{3}: \mathrm{Yb}, \mathrm{Er}$ upconversion fluorescent nanocrystals via a solid-liquid two-phase approach. Scripta Mater. 2008. 58(2): 89-92.

26 Chen C., et al., Ionic Liquid-Based Route to Spherical $\mathrm{NaYF}_{4}$ Nanoclusters with the Assistance of Microwave Radiation and Their Multicolor Upconversion Luminescence. Langmuir 2010. 26(11): 8797-8803.

27 Mi C., et al., Novel microwave-assisted solvothermal synthesis of $\mathrm{NaYF}_{4}: \mathrm{Yb}$, Er upconversion nanoparticles and their application in cancer cell imaging. Langmuir 2011. 27(23): 14632-7.

$28 \mathrm{Li}, \mathrm{F}$., et al., Microwave-assisted synthesis and up-down conversion luminescent properties of multicolor hydrophilic $\mathrm{LaF}_{3}: \mathrm{Ln}^{3+}$ nanocrystals. Dalton Trans 2012.

29 Zhang F., et al., Shape, size, and phase-controlled rare-Earth fluoride nanocrystals with optical up-conversion properties. Chem. Eur. J. 2009. 15(41): 11010-9.

30 Zeng S., et al., High uniformity and monodispersity of sodium rareearth fluoride nanocrystals: controllable synthesis, shape evolution and optical properties. CrystEngComm 2011. 13(5): 1384.

31 Yang T., et al., Cubic sub-20 nm NaLuF 4 -based upconversion nanophosphors for high-contrast bioimaging in different animal species. Biomaterials 2012. 33(14): 3733-42.

32 Liu Q., et al., Sub-10 nm hexagonal lanthanide-doped $\mathrm{NaLuF}_{4}$ upconversion nanocrystals for sensitive bioimaging in vivo. J. Am. Chem. Soc. 2011. 133(43): 17122-5.

33 He M., et al., Dual Phase-Controlled Synthesis of Uniform Lanthanide-Doped $\mathrm{NaGdF}_{4}$ Upconversion Nanocrystals Via an OA/Ionic Liquid Two-Phase System for In Vivo Dual-Modality Imaging. Adv. Funct. Mater. 2011. 21(23): 4470-4477.

$34 \mathrm{He}$ M., et al., A general strategy for the synthesis of upconversion rare earth fluoride nanocrystals via a novel OA/ionic liquid two-phase system. Chem Commun (Camb) 2011. 47(33): 9510-2.

35 Ma J., et al., Folic Acid-Conjugated LaF(3):Yb,Tm@ $\mathrm{SiO}_{2} \mathrm{Nano-}$ probes for Targeting Dual-Modality Imaging of Upconversion Luminescence and X-ray Computed Tomography. J. Phys. Chem. B 
2012. 116(48): 14062-70.

$36 \mathrm{He}, \mathrm{M}$., et al., Phase- and size-controllable synthesis of hexagonal upconversion rare-earth fluoride nanocrystals through an oleic acid/ionic liquid two-phase system. Chem. 2012. 18(19): 5954-69.

37 Pan L., et al., Phase and Size Controllable Synthesis of $\mathrm{NaYbF}_{4}$ Nanocrystals in Oleic Acid/ Ionic Liquid Two-Phase System for Targeted Fluorescent Imaging of Gastric Cancer. Theranostics 2013. 3(3): 210-22

38 Ruitao Chai H.L., Hou Z.Y., Zhang C.M., Peng C., Lin J. Preparation and Characterization of Upconversion Luminescent $\mathrm{NaYF}_{4}: \mathrm{Yb}^{3+}, \mathrm{Er}^{3+}\left(\mathrm{Tm}^{3+}\right) / \mathrm{PMMA}$ Bulk Transparent Nanocomposites Through In Situ Photopolymerization. J. Phys. Chem. C 2010. 114: 610-616.

39 Zeng S., et al., PEG modified $\mathrm{BaGdF}(5): \mathrm{Yb} / \mathrm{Er}$ nanoprobes for multimodal upconversion fluorescent, in vivo X-ray computed tomography and biomagnetic imaging.Biomaterials 2012.33(36):9232-8.

40 Li Z., Zhang Y. Monodisperse Silica-Coated Polyvinylpyrrolidone/

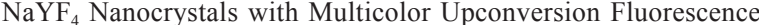
Emission. Angew. Chem. 2006. 118(46): 7896-7899.

41 Dong B., et al., Multifunctional $\mathrm{NaYF}_{4}: \mathrm{Yb}^{3+}, \mathrm{Er}^{3+} @ \mathrm{Ag}$ core/shell nanocomposites: integration of upconversion imaging and photothermal therapy. J. Mater. Chem. 2011. 21(17): 6193.

42 Feng W., Sun L.D., Yan C.H. Ag nanowires enhanced upconversion emission of $\mathrm{NaYF}_{4}: \mathrm{Yb}$,Er nanocrystals via a direct assembly method. Chem Commun (Camb) 2009(29): 4393-5.

43 Liu, N., et al., Highly plasmon-enhanced upconversion emissions from Au@[small beta]-NaYF $\mathrm{N}_{4}: \mathrm{Yb}, \mathrm{Tm}$ hybrid nanostructures. Chem. Commun. 2011. 47(27): 7671-7673.

44 Priyam A., Idris N.M., Zhang Y., Gold nanoshell coated $\mathrm{NaYF}_{4}$ nanoparticles for simultaneously enhanced upconversion fluorescence and darkfield imaging. J. Mater. Chem. 2012. 22(3): 960.

45 Zou W., et al., Broadband dye-sensitized upconversion of nearinfrared light. Nat. Photon. 2012. 6(8): 560-564.

46 Xie X. and X. Liu, Photonics: Upconversion goes broadband. Nat. Mater. 2012. 11(10): 842-3.

47 Idris N.M., et al., Tracking transplanted cells in live animal using upconversion fluorescent nanoparticles. Biomaterials 2009. 30(28): 5104-5113.

48 Cheng L., et al., Highly-sensitive multiplexed in vivo imaging using pegylated upconversion nanoparticles. Nano Res. 2010. 3(10): 722732 .

49 Wang C., et al., Towards whole-body imaging at the single cell level using ultra-sensitive stem cell labeling with oligo-arginine modified upconversion nanoparticles. Biomaterials 2012. 33(19): 4872-81.

50 Sun Y., et al., Radioisotope post-labeling upconversion nanophosphors for in vivo quantitative tracking. Biomaterials 2013. 34(9): 2289-2295.

51 Gao G., et al., One-pot hydrothermal synthesis of lanthanide ions doped one-dimensional upconversion submicrocrystals and their potential application in vivo CT imaging. Nanoscale 2012. 5(1): 351-362.

52 Louie A., Multimodality Imaging Probes: Design and Challenges. Chem. Rev. 2010. 110(5): 3146-3195.

53 Cheng L., et al., Facile preparation of multifunctional upconversion nanoprobes for multimodal imaging and dual-targeted photothermal therapy. Angew. Chem. Int. Ed. 2011. 50(32): 7385-90.

$54 \mathrm{Zhu} \mathrm{X}$., et al., Core-shell $\mathrm{Fe}_{3} \mathrm{O}_{4} @ \mathrm{NaLuF}_{4}: \mathrm{Yb}, \mathrm{Er} / \mathrm{Tm}$ nanostructure for MRI, CT and upconversion luminescence tri-modality imaging. Biomaterials 2012. 33(18): 4618-27.

55 Guo H., et al., Seed-mediated synthesis of $\mathrm{NaY} \mathrm{F}_{4}: \mathrm{Yb}, \mathrm{Er} / \mathrm{NaGdF}_{4}$ nanocrystals with improved upconversion fluorescence and MR relaxivity. Nanotechnology 2010. 21(12): 125602.

56 Ryu J., et al., Facile Synthesis of Ultrasmall and Hexagonal $\mathrm{NaGdF}_{4}$ : $\mathrm{Yb}^{3+}, \mathrm{Er}^{3+}$ Nanoparticles with Magnetic and Upconversion Imaging Properties. J. Phys. Chem. C 2010. 114(49): 21077-21082.

57 Zeng S., et al., Bi-functional $\mathrm{NaLuF}_{4}: \mathrm{Gd}^{3+} / \mathrm{Yb}^{3+} / \mathrm{Tm}^{3+}$ nanocrystals structure controlled synthesis, near-infrared upconversion emission and tunable magnetic properties. J. Mater. Chem. 2012. 22(19): 9870

58 Wang C., Cheng L., Liu Z., Drug delivery with upconversion nanoparticles for multi-functional targeted cancer cell imaging and therapy. Biomaterials 2011. 32(4): 1110-1120.

$59 \mathrm{Xu} \mathrm{H}$., et al., Polymer encapsulated upconversion nanoparticle/iron oxide nanocomposites for multimodal imaging and magnetic targeted drug delivery. Biomaterials 2011. 32(35): 9364-9373.

60 Tian G., et al., $\mathrm{Mn}^{2+}$ dopant-controlled synthesis of $\mathrm{NaYF}_{4}: \mathrm{Yb}$ /Er upconversion nanoparticle for in vivo imaging and drug delivery. Adv Mater 2012. 24(9): 1226-1231

61 Yang D., et al., Hollow structured upconversion luminescent $\mathrm{NaYF}_{4}: \mathrm{Yb}^{3+}, \mathrm{Er}^{3+}$ nanospheres for cell imaging and targeted anticancer drug delivery. Biomaterials 2013. 34(5): 1601-1612.

62 Xiao Q., et al., Novel multifunctional $\mathrm{NaYF}_{4}: \mathrm{Er}^{3+}, \mathrm{Yb}^{3+} / \mathrm{PEGDA}$ hybrid microspheres: NIR-light-activated photopolymerization and drug delivery. Chem. Commun. 2013. 49(15): 1527-1529.

63 Yang Y., et al., Fabrication of and Drug Delivery by an Upconversion Emission Nanocomposite with Monodisperse $\mathrm{LaF}_{3}: \mathrm{Yb}, \mathrm{Er}$ Core Mesoporous Silica Shell Structure. European J. Inorg. Chem. 2010. 2010(33): 5195-5199.

64 Tian G., et al., Facile Fabrication of Rare-Earth-Doped $\mathrm{Gd}_{2} \mathrm{O}_{3}$ Hollow Spheres with Upconversion Luminescence, Magnetic Resonance, and Drug Delivery Properties. J. Phys. Chem. C 2011.115 (48): 23790-23796

65 Li, L.-L., et al., An Exceptionally Simple Strategy for DNAFunctionalized Up-Conversion Nanoparticles as Biocompatible Agents for Nanoassembly, DNA Delivery, and Imaging. J. Am. Chem. Soc. 2013. 135(7): 2411-2414.

66 Yang Y., et al., NIR light controlled photorelease of siRNA and its targeted intracellular delivery based on upconversion nanoparticles.Nanoscale 2013. 5(1): 231-238.

67 Zhang F., et al., Mesoporous multifunctional upconversion luminescent and magnetic "nanorattle" materials for targeted chemotherapy. Nano lett. 2011. 12(1): 61-67.

68 Cheng L., Wang C., Liu Z., Upconversion nanoparticles and their composite nanostructures for biomedical imaging and cancer therapy. Nanoscale 2012. 5(1):23-37.

69 Zhang P., et al., Versatile Photosensitizers for Photodynamic Therapy at Infrared Excitation. J. Am. Chem. Soc. 2007. 129(15): 4526-4527.

70 Chatterjee D.K., Z. Yong, Upconverting nanoparticles as nanotransducers for photodynamic therapy in cancer cells. Nanomedicine 2008. 3(1): 73-82.

71 Qian H.S., et al., Mesoporous-silica-coated up-conversion fluorescent nanoparticles for photodynamic therapy. Small 2009.5(20):2285-90.

72 Idris N.M., et al., In vivo photodynamic therapy using upconversion nanoparticles as remote-controlled nanotransducers. Nat. Med. 2012. 18(10): 1580-5.

73 Park.Y.I., et al., Theranostic Probe Based on Lanthanide-Doped Nanoparticles for Simultaneous In Vivo Dual-Modal Imaging and Photodynamic Therapy. Adv. Mater. 2012. 24(42): 5755-5761.

74 Qian L., et al., Gold decorated NaYF4:Yb,Er/NaYF ${ }_{4} /$ silica (core/shell/shell)upconversion nanoparticles for photothermal destruction of BE(2)-C neuroblastoma cells. J. Nanopart. Res. 2011. 13(2): 499-510.

75 Dong B., et al., Multifunctional $\mathrm{NaYF}_{4}: \mathrm{Yb}^{3+}, \mathrm{Er}^{3+} @ \mathrm{Ag}$ core/shell nanocomposites: integration of upconversion imaging and photothermal therapy. J. Mater. Chem. 2011. 21(17): 6193-6200.

76 Cheng L., et al., Facile Preparation of Multifunctional Upconversion Nanoprobes for Multimodal Imaging and Dual-Targeted Photothermal Therapy. Angew. Chem. 2011. 123(32): 7523-7528.

77 Cheng L., et al., Multifunctional nanoparticles for upconversion luminescence/MR multimodal imaging and magnetically targeted photothermal therapy. Biomaterials 2012. 33(7): 2215-2222.

Copyright:(c) 2013 N. Zhou et al. This is an open-access article distributed under the terms of the Creative Commons Attribution License, which permits unrestricted use, distribution, and reproduction in any medium, provided the original author and source are credited. 